\title{
Illumination effects in face recognition
}

\author{
WENDY L. BRAJE \\ St. Cloud State University, St. Cloud, Minnesota \\ DANIEL KERSTEN \\ University of Minnesota, Minneapolis, Minnesota \\ MICHAEL J. TARR \\ Brown University, Providence, Rhode Island \\ and \\ NIKOLAUS F. TROJE \\ Queen's University, Kingston, Ontario, Canada
}

\begin{abstract}
How do observers recognize faces despite dramatic image variations that arise from changes in illumination? This paper examines (1) whether face recognition is sensitive to illumination direction and (2) whether cast shadows improve performance by providing information about illumination or hinder performance by introducing spurious edges. In Experiment 1, observers judged whether two sequentially presented faces, illuminated from the same direction or different directions, were the same individual or not. Cast shadows were present for half of the observers. Performance was impaired by a change in the illumination direction and by the presence of shadows. In Experiment 2, observers learned to name eight faces under one illumination direction (left/right) and one cast-shadow condition (present/absent); they were later tested under novel illumination and shadow conditions. Performance declined for unfamiliar illumination directions, but not for unfamiliar shadow conditions. The finding that face recognition is illumination dependent is consistent with the use of image-based representations. The results indicate that face recognition processes are sensitive to either the direction of lighting or the resultant pattern of shading, and that cast shadows can hinder recognition, possibly by masking informative features or leading to spurious contours.
\end{abstract}

Illumination variation has enormously complex effects on the image of an object. In the image of a familiar face, changing the direction of illumination leads to shifts in the location and shape of shadows, changes in highlights, and reversal of contrast gradients. Yet everyday experience shows that we are remarkably good at recognizing faces despite such variations in lighting. In this study, we examined how humans recognize faces, given image variations caused by changes in lighting direction and by cast shadows. One issue was whether faces are represented in an illumination-invariant or illumination-dependent manner. A second issue was whether cast shadows improve face recognition by providing information about surface shape and illumination direction or hinder performance by introducing spurious edges that must be discounted prior to recognition. The influences of illumination direction and cast shadows were examined using both short- and long-term memory paradigms.

This research was supported by grants from the National Science Foundation (SBR-9631682), National Institutes of Health (EY02857), Air Force Office of Scientific Research (F49620-91-J-0169), NATO, and the Max-Planck-Institut für biologische Kybernetik. Correspondence should be addressed to W. L. Braje, Department of Psychology, St. Cloud State University, 304 Whitney House, St. Cloud, MN, 56301 (e-mail:wbraje@stcloudstate.edu).
The large image variations that result from changing the illumination direction have been demonstrated by Adini, Moses, and Ullman (1995). They compared images of several faces rendered with the same lighting direction or different lighting directions. Several representations of these images were considered: gray-scale images, images filtered with Gabor functions, edge maps, and first and second derivatives of gray-scale images. For all of these representations, they found that varying the illumination direction resulted in larger image differences than did varying the identity of the face.

How can we recognize faces given these dramatic image variations that arise from changes in illumination? One class of models proposes that, in the early stages of processing, the visual system extracts features that are invariant over changes in illumination (e.g., edges defined by surface material, orientation discontinuities, or occlusion) and discounts spurious features (e.g., shadows and specularities; Biederman, 1987; Biederman \& Ju, 1988; Marr $\&$ Nishihara, 1978). These edge-based models presume that the early image representation is much like an artist's line drawing, with lines marking key surface shapes and material features.

According to such models, recognition should be unaffected by changes in lighting conditions. Consistent with this view, Moses, Ullman, and Edelman (1996) demon- 
strated that observers were highly accurate at naming pictures of faces under novel illumination conditions. Additionally, neurophysiological studies have shown that some neurons responsive to faces are invariant to changes in the viewpoint, position, spatial frequency, and/or size of the object (Ito, Tamura, Fujita, \& Tanaka, 1995; Rolls, 1992; Rolls, Baylis, \& Leonard, 1985; Tovee, Rolls, \& Azzopardi, 1994). It might be expected that neurons of this type would also be insensitive to changes in illumination conditions. From a computational perspective, however, no algorithm has yet been devised that can produce a clean line drawing of natural objects and smooth objects (such as faces) under all the image variations that occur naturally (see Adini et al., 1995).

Image-based models, on the other hand, propose that object representations are more closely tied to the twodimensional (2-D) image (Bülthoff, Edelman, \& Tarr, 1995; Gauthier \& Tarr, 1997; Poggio \& Edelman, 1990). According to these models, introducing a new illumination direction results in a large change in the stimulus representation, which should lead to an impairment of recognition. Cavanagh (1991) has pointed out that cast shadows are difficult to identify using early mechanisms. This suggests that it is easier to encode illumination effects than to discount them. Similarly, Adini et al. (1995) and Ullman (1996, p. 324) have argued that illumination must be processed using higher level mechanisms. Moreover, illumination information may be useful for computing three-dimensional (3-D) shape and identifying shadows (Tarr, Kersten, \& Bülthoff, 1998). Thus, illumination information might be retained in face representations because it is too difficult to discount at an early level and/or because it is useful at higher levels.

Support for image-based models comes from recent studies showing that changing the direction of illumination can influence recognition of geometric objects (Tarr et al., 1998) and facial surfaces (i.e., 3-D renderings of faces without color or texture; Hill \& Bruce, 1996; Troje \& Bülthoff, 1998). It has also been shown that faces are more difficult to recognize when presented in photographic negative, implying that edge information, without contrast polarity information, is not sufficient to account for face recognition (Hayes, Morrone, \& Burr, 1986). Other studies show that the direction of illumination can influence 3-D shape perception (Belhumeur, 1997; Berbaum, Bever, \& Chung, 1983; Christou \& Koenderink, 1996). Furthermore, although Moses et al. (1996) found high accuracy for naming pictures of faces under novel illumination conditions, their observers did show a cost in reaction time ( $\mathrm{RT})$.

Neurophysiological studies also suggest that some effects of illumination may be encoded. Warrington (1982) described patients with right posterior lesions who had difficulty recognizing objects under different illumination conditions. Weiskrantz (1990) discussed similar findings in monkeys with lesions in inferotemporal and prestriate areas. Hietanen, Perrett, Oram, Benson, and Dittrich (1992) discovered face-selective cells in the temporal cortex of the macaque that were sensitive to changes in illumination. Such findings suggest that information about illumination (or image effects resulting from illumination) is not discarded but is instead retained in the object representation.

One consequence of changing illumination conditions is a change in the characteristics (e.g., shape and location) of shadows. How do shadows affect face recognition? Edge-based models of object recognition propose that the visual system discounts spurious features, such as shadows. In this scheme, shadows should not affect recognition. Support for this view was found by Braje, Legge, and Kersten (1998). Their observers named pictures of familiar natural objects (fruits and vegetables), shown with or without shadows. They found that shadows had no effect on observers' accuracy or RT.

According to image-based models, however, object representations retain information present in the original image, including shadows. Cavanagh (1991) proposed that early processing of an image involves a crude match of the image to a memory representation, in which all image contours (including shadow contours) are used. Only once a candidate object is selected are the contours labeled as belonging to objects or shadows. If shadows are encoded as part of the object representation, they can be problematic for recognition in that they introduce spurious luminance edges that can be confused with object contours. Consistent with this prediction, Warrington (1982) demonstrated that patients with right posterior lesions had difficulty recognizing photographs of common objects containing shadows, and Moore and Cavanagh (1998) showed that two-tone images of novel objects with shadows are difficult to recognize. Alternatively, encoding shadows might improve recognition by providing useful information about object shape (e.g., surface orientation and curvature) and about illumination conditions in a scene (e.g., light source direction). Tarr et al. (1998) demonstrated that cast shadows can improve recognition of novel geometric objects, suggesting that shadows provide useful information about shape or lighting conditions.

Shadows can be classified into two types: attached shadows and cast shadows. Each type places different requirements on a recognition system attempting to discount shadows at any stage of processing. An attached shadow occurs when a surface gradually turns away from the lighting direction. If a Lambertian shading model is assumed, then the intensity at a given pixel in an attached shadow depends only on the local surface orientation with respect to the illumination direction. Thus, any algorithm (artificial or biological) attempting to discount attached shadows can work locally. A cast shadow occurs when an object is interposed between a light source and a surface, blocking the illumination from reaching the surface (Beck, 1972). Cast shadows may be particularly difficult to dis- 
count, in that they are influenced both by local surface characteristics and by surfaces more distant to the shadowed area.

Face recognition may be particularly susceptible to the influence of shadows. Although Braje et al. (1998) found no effect of shadows on the ability to name familiar natural objects, familiar stimuli may be more resistant to noise in general and to shadows in particular. Faces, on the other hand, although familiar as a class of objects, are not necessarily familiar on an individual basis. They may therefore be less resistant to the influence of shadows. A second reason that shadows should affect face recognition is that fine discrimination is required to distinguish between different faces. Different faces are fairly similar in their global shape, hue, and texture. Because of the small differences between faces, recognizing a particular face requires a detailed analysis, and it may also rely more heavily on correct labeling of contours. Under such conditions, shadows should have a larger impact on recognition.

The present experiments investigated whether or not face representations are illumination dependent and how cast shadows influence face recognition. Experiment 1 used a same/different matching task to examine whether face representations in short-term memory retain illumination and shadow information. Experiment 2 extended the findings to representations in long-term memory by using a naming task. The key issues were whether recognition performance is influenced by (1) changing the direction of illumination and (2) the presence of cast shadows.

\section{EXPERIMENT 1 Matching}

In Experiment 1, observers viewed two sequentially presented faces and decided whether they were the same person or not. The two faces were illuminated from the same direction or different directions on each trial. If face representations are closely tied to the 2-D image, then performance should decline when the illumination changes between the two images. However, if a more invariant encoding is used, illumination should have no effect on performance.

Cast shadows were present for half of the observers and absent for the other half. If cast shadows are retained in the face representation, then they should influence performance in one of two ways. Their presence should lead to an overall decline in performance if they introduce confusing contours or mask important features. If, however, cast shadows provide useful information regarding 3-D shape or illumination, performance should improve when they are present.

\section{Method}

Observers. Thirty-two undergraduate psychology students (17-34 years of age) at the University of Minnesota participated in the experiment for class credit. All had normal or corrected-to-normal visual acuity (Snellen acuity of 20/20 or better) and gave informed consent. The observers were not familiar with the people whose faces were used as stimuli.

Stimuli and Apparatus. Face images were obtained from 3-D models of 80 real human heads (Troje \& Bülthoff, 1996). The colored texture and 3-D geometry of the heads were digitized using a Cyberware 3-D laser scanner. The scanner samples a regular grid of surface points in cylindrical coordinates. Each sample contains the spatial coordinates of the surface point (azimuth, height, and radius), as well as its color (RGB) values. The distance between neighboring points is $0.8^{\circ}$ (roughly $1 \mathrm{~mm}$ ) in the azimutal direction, $0.615 \mathrm{~mm}$ in the vertical direction, and $0.016 \mathrm{~mm}$ along the radius. There were 40 males and 40 females, all Caucasian people between about 20 and 40 years of age. They had no spectacles or facial hair, and they had neutral facial expressions. The hair and back of the head were removed from the 3-D models before the images were created.

The face images were rendered in color in orthographic projection using Wavefront's "The Advanced Visualizer 4.1" (Santa Barbara, CA) on a Silicon Graphics workstation. Each face was rendered from two different viewpoints $\left(7^{\circ}\right.$ and $11^{\circ}$ with respect to the frontal view) and in two sizes $\left(7.9^{\circ} \times 9.5^{\circ}\right.$, and $\left.8.5^{\circ} \times 10.3^{\circ}\right)$, for reasons discussed in the Procedure section below. Faces were illuminated by a point source located $130 \mathrm{~cm}$ from the face. The source was positioned $45^{\circ}$ above and $45^{\circ}$ to the right or left of the viewing axis. The faces were rendered with ray-tracing (producing cast shadows) and without ray-tracing (no cast shadows). A small amount of ambient light was also present, such that the shadowed regions were not entirely dark. Figure 1 illustrates the different renderings. Note that an image with no cast shadows does contain attached shadows and that all cast shadows are intrinsic (i.e., cast by parts of the face onto the face itself).

The experiment was run on an Apple Macintosh Quadra 950 using RSVP software (Williams \& Tarr, 1998). The faces were presented on a black background $\left(<1 \mathrm{~cd} / \mathrm{m}^{2}\right)$ on a $640 \times 480$ pixel Apple 17-in. color monitor. They were roughly 150 pixels horizontally $\times 215$ pixels vertically and were viewed from a distance of $45 \mathrm{~cm}$. The luminance of each face ranged from less than $1 \mathrm{~cd} / \mathrm{m}^{2}$ to $40 \mathrm{~cd} / \mathrm{m}^{2}$.

A $256 \times 256$ pixel mask was created using Adobe Photoshop. The mask consisted of a collage of scrambled face features-eyes, noses, mouths, and ears - taken from the different renderings of the 80 faces. The luminance of the mask was $10 \mathrm{~cd} / \mathrm{m}^{2}$.

Procedure. A sequential matching paradigm was used (see Figure 2). On each trial, a fixation cross was presented for $500 \mathrm{msec}$, followed by a face for $200 \mathrm{msec}$, the mask for $750 \mathrm{msec}$, a second face for $100 \mathrm{msec}$, and the mask again for $500 \mathrm{msec}$.

The observer's task was to decide whether the two faces were the same person or not and to respond by pressing one of two keys on a keyboard. Feedback was not provided. The durations were chosen on the basis of an experiment by Ellis and Allport (1986), who showed that an interstimulus interval of $750 \mathrm{msec}$ was long enough to prevent observers from simply using an iconic representation of the first face to perform the match. With the timing used here, observers are likely to encode the first image in short-term memory for subsequent match to the second image (Tarr et al., 1998). The presentation durations were also chosen to prevent the observers from obtaining ceiling performance (i.e., 100\% accuracy).

RT was measured as the time between the onset of the second face and the observer's keypress. The observers were allowed up to $5 \mathrm{sec}$ to respond. Sensitivity $\left(d^{\prime}\right)$ was calculated using the $z$ scores for the correct responses on same-face trials (hits) and the incorrect responses on different-face trials (false alarms) ${ }^{1}: d^{\prime}=z_{\text {hit }}-z_{\mathrm{fa}}$.

On each trial, the two faces could be illuminated from either the same direction or different directions. Half of the observers viewed faces with cast shadows; the other half viewed faces without cast 


\section{Illumination from left}
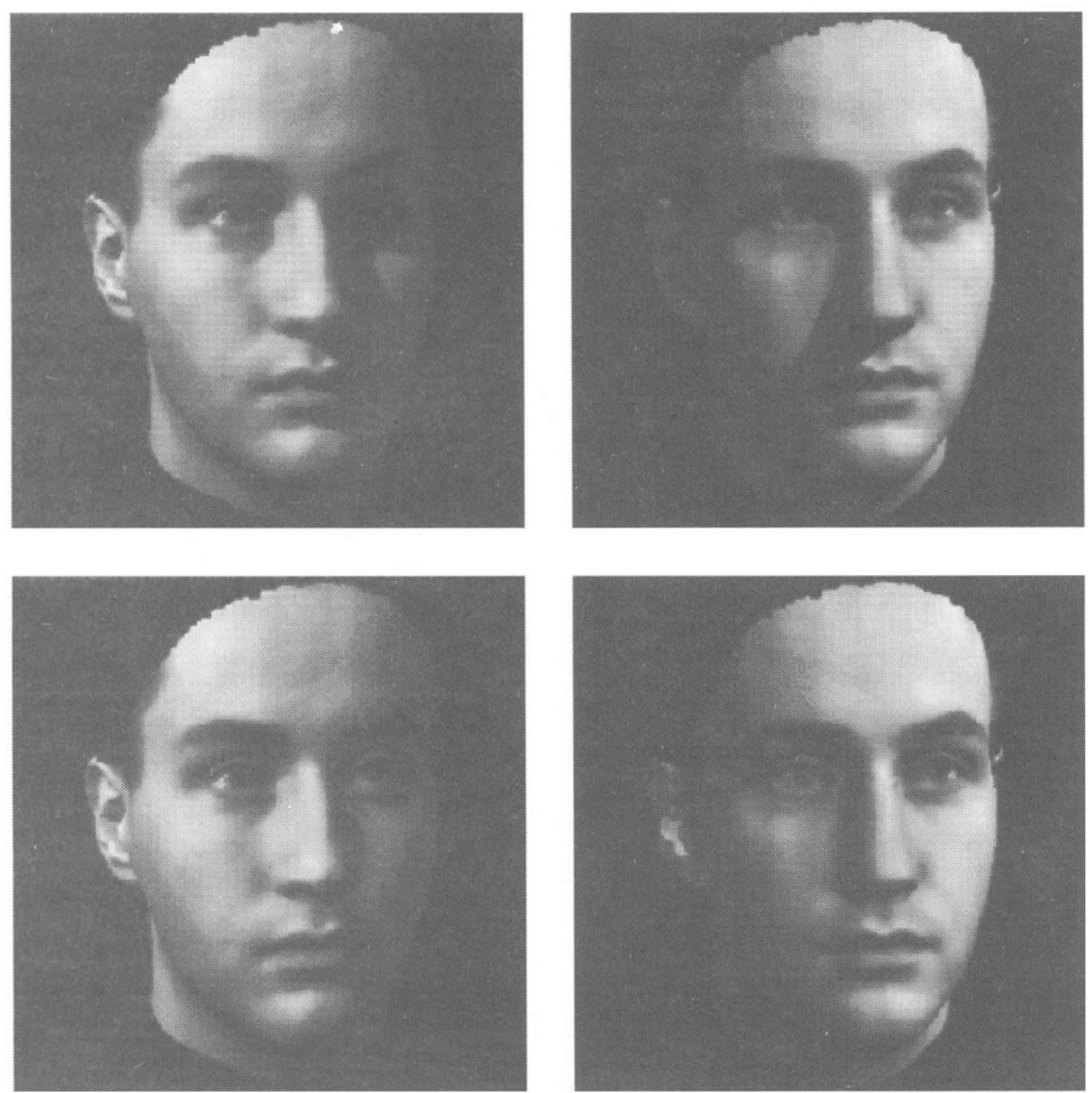

Figure 1. A face in the four rendering conditions (left/right illumination, with/without cast shadows). The viewpoint is $11^{\circ}$ with respect to the frontal view. shadows. The presence or absence of cast shadows was run as a between-groups variable in order to prevent the observers from relying on a salient shadow when making a matching judgment.

Each observer completed a 20 -trial practice block, followed by four blocks of 120 experimental trials. In each block, there were equal numbers of left-illuminated and right-illuminated faces and equal numbers of same-face trials and different-face trials. Over the 480 total trials, each of the 80 possible same-face pairs was presented three times, and a random selection of the 6,320 possible different-face pairs was presented. Each different-face pair was pre-

\section{Illumination from right}

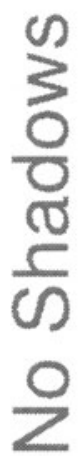

sented only once to each observer. Both same-sex and different-sex pairs were used on different-face trials. An analysis of variance (ANOVA) run using just the trials containing same-sex pairs revealed the same results as found when all trials (both same- and different-sex) were used. Thus, all trials were included in the analyses reported below.

For each presentation of each image, the viewpoint and size were randomly chosen from among those described in the Stimuli section. This prevented the observers from simply matching images for size, silhouette, or local image features. Nonfrontal views were 


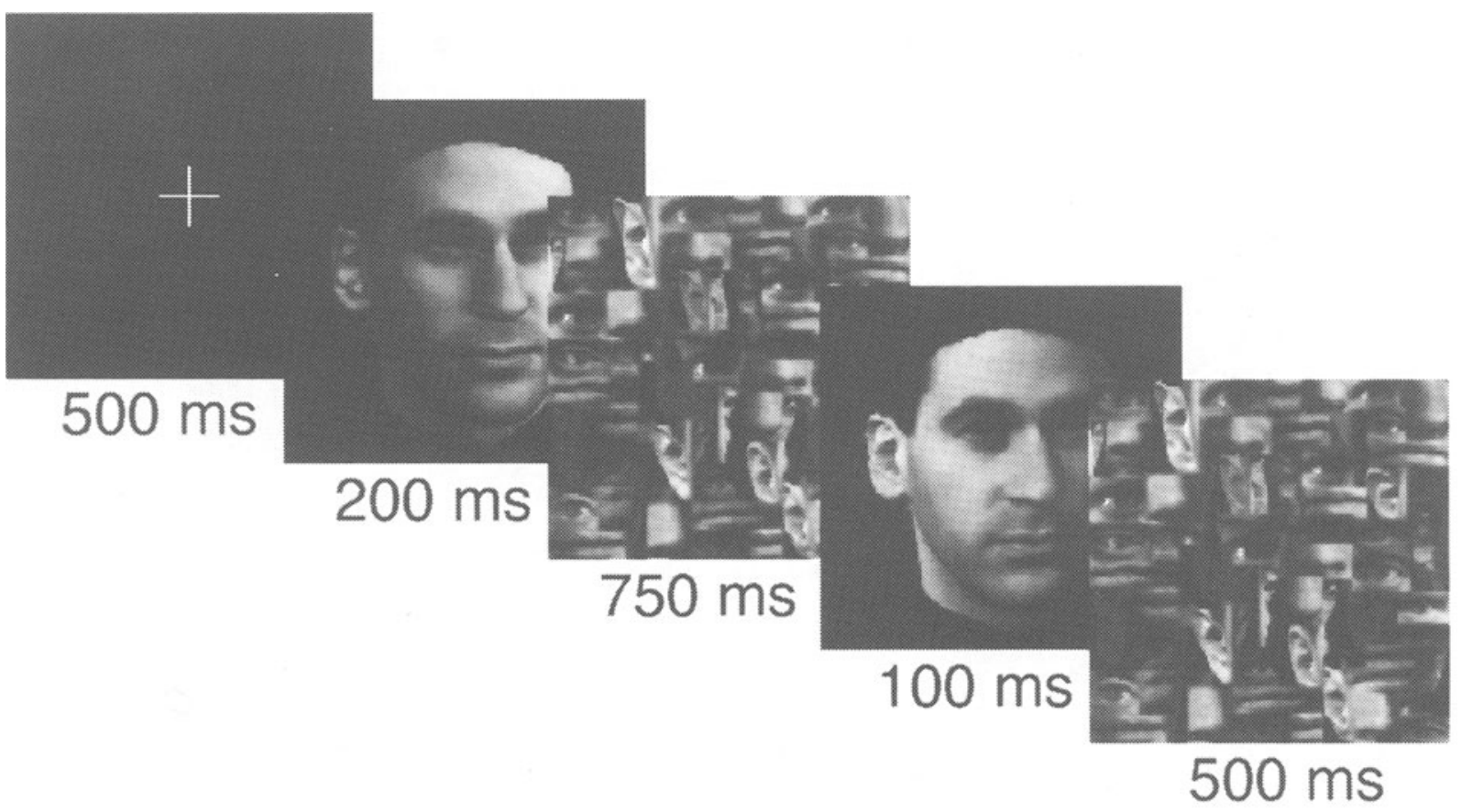

\section{TIME}

Figure 2. Sequential matching paradigm used in Experiment 1. The observers' task was to decide whether the two faces were the same person or not.

used in order to avoid symmetrical image pairs, which would occur in a frontal view when the light source was moved from one side to the other. Symmetrical images are perceptually very similar (Troje \& Bülthoff, 1998) and would produce an accidental situation in which changing the illuminant's position would not influence recognition performance.

\section{Results}

Performance declined when there was a change in illumination direction. Figure 3 shows that, in same-face trials, response time was $44 \mathrm{msec}$ slower when there was a change in illumination direction than when there was no change. In different-face trials (data not shown), changing the illumination direction had no effect on RT. An ANOVA (run on the median RTs for the trials in which a correct response was made) confirmed a main effect of illumination direction $[F(1,30)=18.24, p<.001]$ and an interaction between illumination direction and face (same vs. different) $[F(1,30)=17.98, p<.001]$. A Tukey HSD test $(\alpha=.05)$ confirmed that the illumination effect occurred only for same-face trials. Sensitivity was also lower when there was a change in illumination direction $\left(d^{\prime}=2.3\right)$ than when there was no change $\left(d^{\prime}=2.6\right)$ $[F(1,30)=17.81, p<.001]$. The observers scored $85 \%$ correct when there was a change in illumination and $92 \%$ correct when there was no change.

The presence of cast shadows impaired RT $[F(1,30)=$ $5.54, p<.05]$, but not sensitivity $[F(1,30)=1.81, p=$
.189]. RT was $127 \mathrm{msec}$ slower when cast shadows were present. A Tukey HSD test $(\alpha=.01)$ confirmed that the observers were always slower when cast shadows were present, for all illumination and face conditions.

This decline in performance in images containing cast shadows was not simply a result of lower mean luminance or higher contrast in the cast shadow images. There was no correlation between RT and mean pixel value of each face $\left(R^{2}=.002\right)$ or between RT and RMS contrast $\left(R^{2}=.01\right) .^{2}$

Finally, the effect of illumination was not influenced by the presence of cast shadows: There was no significant interaction between illumination (same vs. different) and cast shadows (present vs. absent) for either RT $[F(1,30)=$ $3.32, p=.078]$ or sensitivity $[F(1,30)=1.62, p=.213]$.

\section{Discussion}

Performance declined when the illumination changed and when cast shadows were present. This suggests that short-term face representations, lasting at least several hundred milliseconds, preserve image information, such as illumination and cast shadows, and it supports imagebased models of recognition. The findings are consistent with the results of Tarr et al. (1998) for recognition of novel geometric objects. In a matching task such as the one used here, they found that recognition was $21 \mathrm{msec}$ faster when there was no change in illumination direction than when 

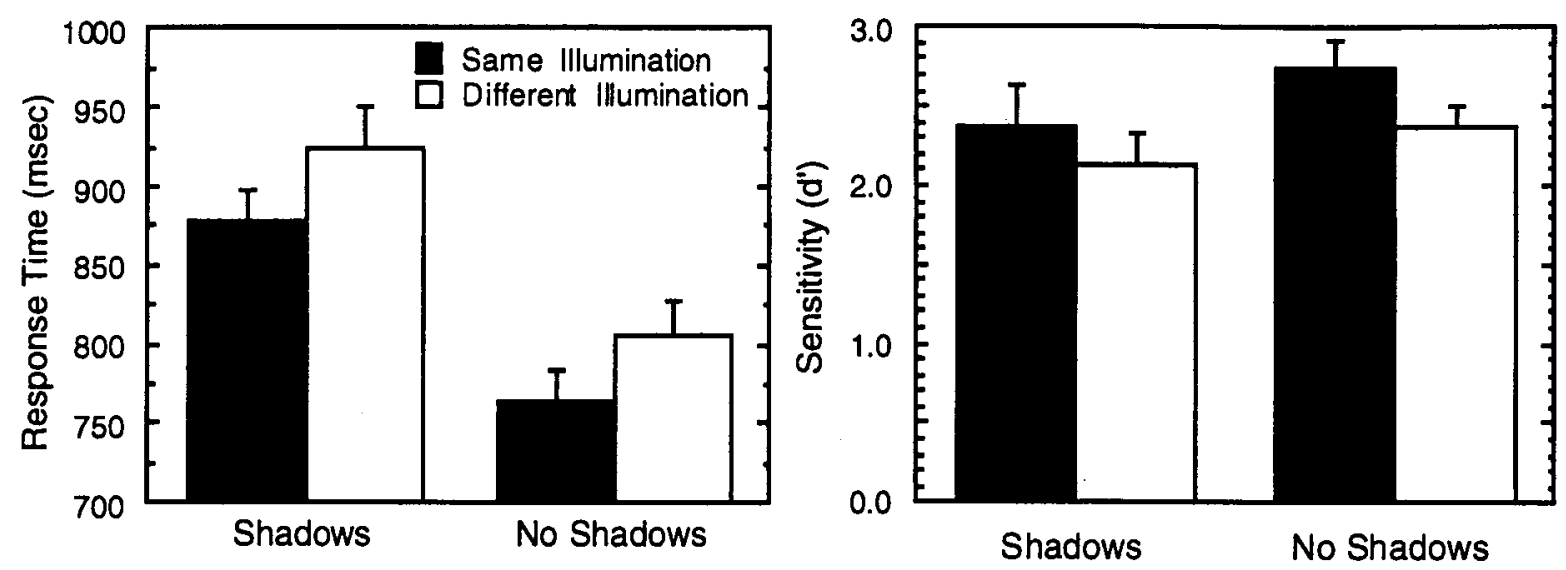

Figure 3. Response time and sensitivity for the trials in which the faces were the same person. Response times shown are the averages of the median response times for trials in which a correct response was made. Error bars show standard errors.

there was a change. In the present study, the difference was $44 \mathrm{msec}$ - even larger than that found by Tarr et al.

One possible reason that changing the illumination impairs performance is that doing so introduces uncertainty into the light position. If the location of the illuminant is ambiguous, it could be more difficult for observers to discount the illuminant. Cast shadows can provide information about the location of the light source. Therefore, when cast shadows are present, changing the illumination direction might be expected to have a smaller effect on recognition. This interaction was not found: The effect of changing the illumination direction was the same whether cast shadows were present or absent. If the lighting was indeed ambiguous, then the observers did not make use of cast shadows to disambiguate it.

The decline in performance in images containing cast shadows could be explained in two ways. One possibility is that time is required for locating and discounting cast shadows (equivalently, higher level processing may be needed). However, the finding that changing the illumination direction impaired performance suggests that image information is preserved, and so it seems unlikely that cast shadows in the image are discounted. A second possibility is that cast shadows mask informative features, forcing the visual system to rely on a smaller (less informative) set of features for recognition. This possibility requires further investigation.

In summary, the results are consistent with the assumption that image information is preserved in short-term face representations. Experiment 2 examined whether these results extend to longer-term representations.

\section{EXPERIMENT 2 Naming}

In Experiment 1, the observers needed to hold the first face in memory for only about $1 \mathrm{sec}$ in order to make a judgment. It is possible that these short-term representations retain image information, while longer-term repre- sentations are more invariant. Experiment 2 therefore examined the effects of cast shadows and illumination on these long-term representations of faces.

It may also be argued that the matching task used in Experiment 1 was not an accurate measure of recognition ability. The observers were asked to detect differences between the stimuli, rather than to explicitly recognize them. In this case, it is possible that illumination-dependent performance arose from within a system that looks for image differences and is sensitive to any changes between stimuli, rather than from within the face-recognition system itself. Thus, a second purpose of Experiment 2 was to examine illumination effects using a more natural facerecognition task.

The observers were trained for 2 days to recognize eight faces under one illumination direction and one castshadow condition. The faces were later presented under new illumination and cast-shadow conditions for observers to recognize. If long-term representations preserve image information, the results should be similar to those in Experiment 1: Performance should be best when the faces are presented under familiar illumination and cast-shadow conditions. Additionally, the overall impairment caused by cast shadows in Experiment 1 might disappear in Experiment 2: The observers should have sufficient time to either build representations based on visible features (if cast shadows mask informative features) or to use higher level mechanisms to process cast shadows.

\section{Methods}

Observers. Twenty University of Minnesota undergraduate psychology students (18-38 years of age) participated in the experiment for class credit. None had participated in Experiment 1. All were Caucasian, had normal or corrected-to-normal visual acuity (Snellen acuity of 20/20 or better), and gave informed consent. The observers were not familiar with the people whose faces were used as stimuli.

Stimuli and Apparatus. The stimuli consisted of eight fullcolor faces ( 4 males and 4 females) presented on a black background. The eight faces were randomly selected from the 80 used 
in Experiment 1. Only the $11^{\circ}$ view and the larger sized images were used. The lighting and cast-shadow conditions were identical to those used in Experiment 1.

The experiment was run on a Power Macintosh 7100 using RSVP software (Williams \& Tarr, 1998). The images were presented on a black background $\left(<1 \mathrm{~cd} / \mathrm{m}^{2}\right)$ on a $640 \times 480$ pixel Apple 17-in. color monitor

Voice-activated RT was recorded using the CMU Button Box (accurate within $1 \mathrm{msec}$ ). The observers' verbal responses (the names of the faces) were compiled by the experimenter using an IBM XT personal computer.

Procedure. The experiment was conducted over 2 consecutive days for each observer. There were three phases to the experiment: training, practice, and testing.

During training, the observers learned to associate a name with each face. For each observer, all faces were illuminated from the same direction (right or left) and were presented in the same castshadow condition (present or absent). Five observers were trained in each of the four lighting and cast-shadow conditions. On each trial, a face was presented for $2 \mathrm{sec}$ with its name written below it, and the observers were instructed to simply learn the name of each face; no response was required. There were three blocks of 16 trials (each face was presented twice per block), and the order of trials was randomized for each observer.

The training was followed by a practice phase, in which the observers practiced naming the faces they had just learned. The faces were shown in the familiar illumination and shadow conditions (i.e., the same as those seen during training). Each trial consisted of a fixation cross for $500 \mathrm{msec}$, followed by a warning tone, and then one of the eight faces. The purpose of the tone was to signal the beginning of a trial to the experimenter, who could not see the images being presented. The task was to name the face aloud as quickly and accurately as possible. The face remained on the screen until the observer responded or $10 \mathrm{sec}$ had passed. After the observer responded, the correct name and the observer's RT were displayed for $500 \mathrm{msec}$. On the ist day of the experiment, the observers completed four blocks of 40 practice trials. Each of the eight faces was presented five times per block. On the 2nd day of the experiment, the observers completed two additional 40 -trial blocks of practice. The observers were required to reach at least $90 \%$ accuracy by the end of the final practice session. ${ }^{3}$

The final phase was the testing phase. The procedure was the same as for the practice trials, except that feedback was not provided. The stimuli consisted of the eight faces in each of the four possible rendering conditions: left/right illumination and with/without cast shad- ows. Eight of these images were familiar (those the observers had trained and practiced on), and the other 24 were unfamiliar (the same eight faces but rendered under the other three illumination/castshadow conditions). The observers completed eight blocks of 32 trials, with each of the 32 images presented once per block. RT was measured as the time between the onset of the face and the onset of the observer's verbal response. ${ }^{4}$

\section{Results}

Accuracy was high (>90\%) in all conditions, confirming that the observers had learned the faces well. Except for an improvement across blocks (see below), no main effects or interactions were significant for the accuracy data. Only the RTs for the testing phase will be considered here. RTs are compared for familiar versus unfamiliar illumination directions and cast-shadow conditions.

Recognition was impaired when faces were displayed under unfamiliar illumination. Median RTs (see Figure 4) were $134 \mathrm{msec}$ slower when the illumination at testing was different from the illumination during training $[F(1,15)=$ $34.53, p<.001] .5$ This difference (a $7 \%$ change) is of similar magnitude to that in Experiment 1 (a $5 \%$ change).

Cast shadows did not influence performance. RTs were equally fast for recognizing faces under familiar and unfamiliar cast-shadow conditions $[F(1,15)=0.90, p=.36$, for the interaction between trained and tested cast-shadow conditions]. The observers trained with no cast shadows tended to respond more quickly overall during the test phase, but this effect did not reach significance $[F(1,15)=$ $2.69, p=.12]$. The presence of cast shadows during the test phase did not affect RT $[F(1,15)=0.21, p=.65]$.

During the testing phase, RT decreased across blocks $[F(7,105)=5.51, p<.001]$ from an average of $1,329 \mathrm{msec}$ in the first block to $885 \mathrm{msec}$ in the final block. Accuracy also improved $[F(7,105)=2.76, p<.02]$ from $92 \%$ to $97 \%$ correct. However, the effects of illumination and cast shadows did not change across blocks: None of the cast-shadow or illumination variables interacted with block number.

\section{Train with shadows}

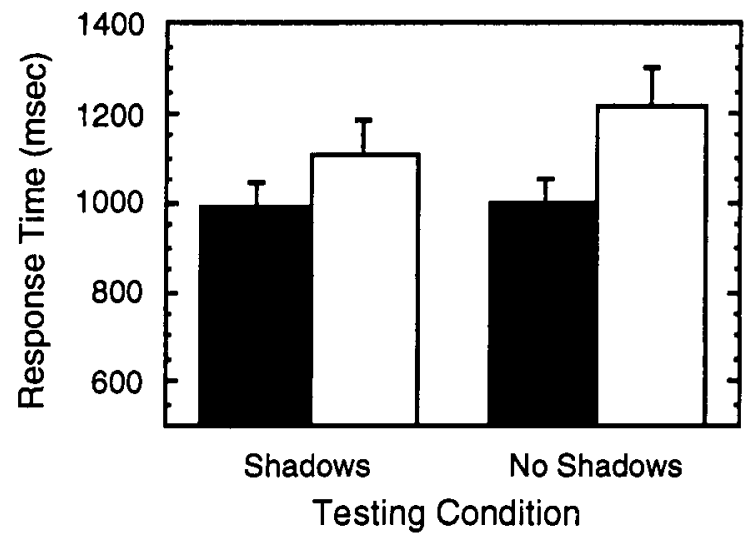

Train with no shadows

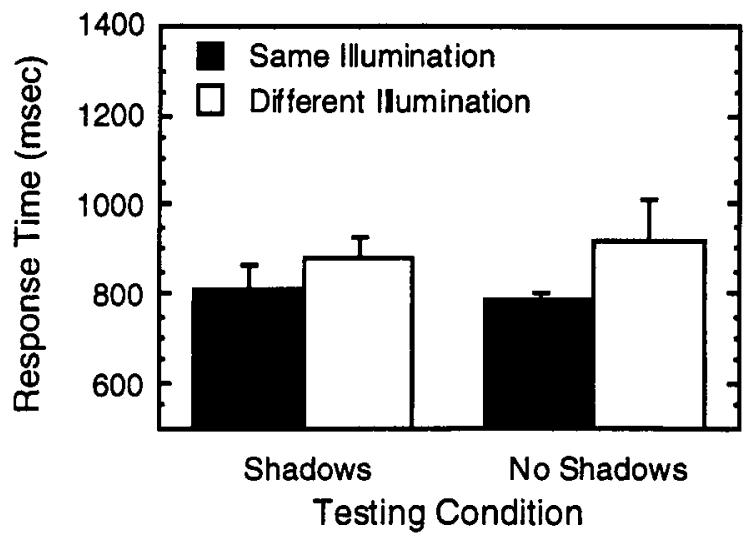

Figure 4. Response times during testing for naming faces under familiar or unfamiliar illumination and cast-shadow conditions. Response times shown are averages of the median response times for trials in which a correct response was made. Error bars show standard errors. 


\section{Discussion}

The results of Experiment 2 partially replicated the results of Experiment 1. The effect of illumination direction was still present when a naming task was used, demonstrating that illumination information is retained even in long-term representations. This finding also suggests that the illumination effects observed in Experiment 1 arose from within a face-recognition system, rather than a system that simply detects image differences. The results of Experiment 2, like those of Experiment 1, are thus consistent with an image-based representation of illumination.

It should be noted that naming tasks have sometimes failed to reveal invariance. For example, Biederman and colleagues have found invariance for translation, reflection, and size using a priming paradigm (Biederman \& Cooper, 1991, 1992; Cooper, Biederman, \& Hummel, 1992; Fiser \& Biederman, 1995). The present results are therefore significant in that they demonstrate the existence of image-based processing using a naming task.

Unlike Experiment 1, Experiment 2 revealed no effect of cast shadows. The observers performed equally well in the testing phase whether they learned the faces with cast shadows present or absent. This demonstrates that a certain amount of invariance with respect to cast shadows is acquired as observers learn to recognize faces. The observers might have had sufficient time to use higher level mechanisms for dealing with cast shadows. Alternatively, if cast shadows were masking important features, the observers may have learned to recognize the faces using other features.

\section{GENERAL DISCUSSION}

Face recognition was found to be sensitive to the presence of cast shadows and to changes in illumination. The observers were slower and less accurate at matching and naming faces when there was a change in illumination direction. There was also a cost in speed and accuracy for matching (but not naming) faces that contained cast shadows.

The finding that illumination changes impair recognition is in agreement with the recent findings of Tarr et al. (1998), and it is consistent with image-based models of face recognition. The findings do contradict some neurophysiological studies, which show that some face-selective cells respond over a wide range of viewpoints, positions, spatial frequencies, and sizes (Ito et al., 1995; Rolls, 1992; Rolls et al., 1985; Tovee et al., 1994). On the other hand, our findings are in agreement with studies that have shown face-selective cells to be sensitive to the viewpoint of faces (Desimone, Albright, Gross, \& Bruce, 1984; Perrett et al., 1991) and, more importantly, to changes in illumination (Hietanen et al., 1992).

Why should the visual system encode illumination information? For one thing, knowledge of illumination conditions is useful for computing 3-D shape. This may be particularly important in face recognition, a task for which the stimuli are geometrically similar. Several studies have demonstrated that viewpoint dependence is stronger when objects are most similar (Edelman, 1995; Tarr \& Bülthoff, 1995), and it is likely that illumination dependence would behave in the same manner. Tarr et al. (1998) have argued that discounting illumination effects increases the similarity between objects, thus making them harder to distinguish. Preserving illumination information may therefore be useful for discriminating between different objects.

Cast shadows were found to impair performance on the matching task (Experiment 1). This is consistent with an image-based model of face recognition, and it suggests that shadows introduce spurious contours that are confused with surface contours. It is also possible that some of the shadows were cast onto features important for recognition, thus making such features difficult to use. A third possibility is that the shadows that are cast onto a particular surface disrupt shape-from-shading processesfor example, by making a curved surface (which would otherwise have a shading gradient) uniformly dark. Further research is needed to distinguish between these possibilities.

The finding that cast shadows impaired performance contradicts Tarr et al.'s (1998) results, which showed that cast shadows improved recognition of geometric objects. One reason for this discrepancy may be that the stimuli used by Tarr et al. were relatively simple compared with faces, and cast shadows may be more likely to obscure details in the more complex face images. Tarr et al. have also argued that the 3-D shape of faces is well known, and so cast shadows are unlikely to offer much additional information. This argument is supported by the finding that cast shadows did not influence performance on the face-naming task.

In the naming task (Experiment 2), the observers were given plenty of opportunities to "learn" the faces. Even if cast shadows normally cause problems in building early face representations, the observers had ample time in the naming experiment to either "solve" the problem of labeling shadow edges or resort to an alternative recognition strategy that is more invariant with respect to shadows.

Further research is necessary in order to test the generality of these results. In particular, the present experiments used a slightly oblique viewpoint $\left(7^{\circ}-11^{\circ}\right)$ for the faces, which may not provide adequate information for generalization across illumination. Troje and Bülthoff (1996) showed that recognition was more viewpoint invariant for faces initially learned from a viewpoint between $22.5^{\circ}$ and $45^{\circ}$ than those learned from a frontal view $\left(0^{\circ}\right)$. Thus, it may be the case that the viewpoints used in the present studies $\left(7^{\circ}\right.$ and $\left.11^{\circ}\right)$ were not optimal for face encoding. However, Tarr et al. (1998) found that illumination dependency in the recognition of geometric objects was not influenced by the familiarity of the view- 
point in which the object was presented. This suggests that the present findings would not change if different viewpoints were used.

Recognition models that rely on edge detection and/or early spatial filtering cannot account for the visual system's ability to allow for illumination variation. One alternative approach is to compute the direction of illumination and then use this to determine 3-D shape in face images (Kersten, O'Toole, Sereno, Knill, \& Anderson, 1987; Pentland, 1990). However, it is unlikely that human vision uses a global estimate of light source direction to compute shape-from-shading or depth-from-shadows (see Tarr et al., 1998, for a discussion). Another approach comes from "appearance-based" models. Here, low-dimensional representations that do not explicitly distinguish shape, material, and lighting are extracted (e.g., through principal components analysis). These models do not explicitly represent illumination but instead store illumination-specific models (Murase \& Nayar, 1995). It is unclear, however, how well these models can deal with novel combinations of illumination and material changes. A third approach is to use a linear model of illumination to derive illumination basis images for a specific view of an object (Epstein, Hallinan, \& Yuille, 1995; Hallinan, 1994, 1995; Shashua, 1992, 1996). These "eigenimages" define the space over which the model can generalize to new illumination conditions (Belhumeur \& Kriegman, 1996). In this model, cast shadows can be treated as residuals, in keeping with Cavanagh's (1991) suggestion that cast shadows are identified by a secondary top-down process.

In summary, the results demonstrated that both shortand long-term face representations are closely tied to information contained in the original image. Face recognition was found to be illumination dependent, indicating that face-recognition processes are sensitive to either the direction of lighting or the resultant pattern of shading. Cast shadows impaired recognition on a sequential matching task, perhaps because they introduce confusing contours. On a long-term task, however, observers are able to overcome the harmful effects of shadows, indicating that some degree of invariance can be achieved.

\section{REFERENCES}

Adini, Y., Moses, Y., \& Ullman, S. (1995). Face recognition: The problem of compensating for changes in illumination direction (Rep. No. CS93-21). Rehovot, Israel: Weizmann Institute of Science.

BECK, J. (1972). Surface color perception. Ithaca, NY: Cornell University Press.

BelHumeur, P. N. (1997). The bas-relief ambiguity. In Proceedings of the IEEE Computer Society Conference on Computer Vision and Pattern Recognition (pp. 1060-1066). Los Alamitos, CA: IEEE Computer Society Press.

Belhumeur, P. [N.], \& Kriegman, D. (1996). What is the set of images of an object under all possible lighting conditions? In Proceedings of the IEEE Computer Society Conference on Computer Vision and Pattern Recognition (pp. 270-277). Los Alamitos, CA: IEEE Computer Society Press.

Berbaum, K., Bever, T., \& Chung, C. S. (1983). Light source position in the perception of object shape. Perception, 12, 411-416.
Biederman, I. (1987). Recognition-by-components: A theory of human image understanding. Psychological Review, 94, 115-147.

Biederman, I. \& COOPER, E. E. (1991). Evidence for complete translational and reflectional invariance in visual object priming. Perception, 20, 585-593.

Biederman, I., \& CoOPER, E. E. (1992). Size invariance in visual object priming. Journal of Experimental Psychology: Human Perception \& Performance, 18, 121-133.

Biederman, I., \& Ju, G. (1988). Surface versus edge-based determinants of visual recognition. Cognitive Psychology, 20, 38-64.

Braje, W. L., LegGe, G. E., \& Kersten, D. (1998). Invariant recognition of natural objects in the presence of shadows. Manuscript submitted for publication.

BülthofF, H. H., Edelman, S. Y., \& TARR, M. J. (1995). How are threedimensional objects represented in the brain? Cerebral Cortex, $\mathbf{5}$, 247-260.

Cavanagh, P. (1991). What's up in top-down processing? In A. Gorea (Eds.), Representations of vision: Trends and tacit assumptions in vision research (pp. 295-304). Cambridge: Cambridge University Press.

Christou, C. G., \& Koenderink, J. J. (1996). Shape from shading and shape constancy under variations in illumination. Investigative Ophthalmology \& Visual Science, 37, S934.

Cooper, E. E., Biederman, I., \& Hummel, J. E. (1992). Metric invariance in object recognition: A review and further evidence. Canadian Journal of Psychology, 46, 191-214.

Desimone, R., Albright, T. D., Gross, C. G., \& Bruce, C. (1984). Stimulus-selective properties of inferior temporal neurons in the macaque. Journal of Neuroscience, 4, 2051-2062.

Edelman, S. (1995). Class similarity and viewpoint invariance in the recognition of 3D objects. Biological Cybernetics, 72, 207-220.

Ellis, R., \& All.PORT, D. A. (1986). Multiple levels of representation for visual objects: A behavioural study. In A. G. Cohn \& J. R. Thomas (Eds.), Artificial intelligence and its applications (pp. 245-257). New York: Wiley.

Epstein, R., Hallinan, P. W., \& Yuille, A. L. (1995). $5 \pm$ eigenimages suffice: An empirical investigation of low-dimensional lighting models. In IEEE Workshop on Physics-Based Modeling in Computer Vision (pp. 108-116). Los Alamitos, CA: IEEE Computer Society Press.

Fiser, J., \& Biederman, I. (1995). Size invariance in visual object priming of gray-scale images. Perception, 24, 741-748.

GAUTHIER, I., \& TARR, M. J. (1997). Becoming a "greeble" expert: Exploring mechanisms for face recognition. Vision Research, 37, 16731682.

Hallinan, P. W. (1994). A low-dimensional lighting representation of human faces for arbitrary lighting conditions. In Proceedings of the IEEE Computer Society Conference on Computer Vision and Pattern Recognition (pp. 995-999). Los Alamitos, CA: IEEE Computer Society Press.

HALlinan, P. W. (1995). A deformable model for face recognition under arbitrary lighting conditions. Unpublished doctoral dissertation, Division of Applied Sciences, Harvard University.

Hayes, T., Morrone, M. C., \& Burr, D. C. (1986). Recognition of positive and negative bandpass-filtered images. Perception, 15, 595-602.

Hietanen, J. K., Perrett, D. I., Oram, M. W., Benson, P. J., \& DittRICH, W. H. (1992). The effects of lighting conditions on responses of cells selective for face views in the macaque temporal cortex. Experimental Brain Research, 89, 157-171.

HiLl, H., \& BruCE, V. (1996). Effects of lighting on the perception of facial surfaces. Journal of Experimental Psychology: Human Perception \& Performance, 22, 986-1004.

Ito, M., Tamura, H., Fujita, I., \& Tanaka, K. (1995). Size and position invariance of neuronal responses in monkey inferotemporal cortex. Journal of Neurophysiology, 73, 218-226.

Kersten, D., O’Toole, A. J., Sereno, M. E., Knill, D. C., \& AnderSON, J. A. (1987). Associative learning of scene parameters from images. Applied Optics, 26, 4999-5006.

Macmillan, N. A., \& Creelman, C. D. (1991). Detection theory: A user's guide. New York: Cambridge University Press.

MARR, D., \& NishihaRA, H. K. (1978). Representation and recognition 
of the spatial organization of three-dimensional shapes. Philosophical Transactions of the Royal Society of London: Series B, 200, 269-294.

Moore, C., \& Cavanagh, P. (1998). Recovery of 3D volume from 2tone images of novel objects. Cognition, 67, 45-71.

Moses, Y., Ullman, S., \& Edelman, S. (1996). Generalization to novel images in upright and inverted faces. Perception, 25, 443-462.

MURASE, H., \& NAYAR, S. (1995). Visual learning and recognition of 3-D objects from appearance. International Journal of Computer Vision, 14, 5-24.

PENTLAND, A. P. (1990). Linear shape from shading. International Journal of Computer Vision, 4, 153-162.

Perrett, D. I., Oram, M. W., Harries, M. H., Bevan, R., Hietanen, J. K., \& BENSON, P. J. (1991). Viewer-centred and object-centred coding of heads in the macaque temporal cortex. Experimental Brain Research, 86, 159-173.

Poggio, T., \& Edelman, S. (1990). A network that learns to recognize three-dimensional objects. Nature, 343, 263-266.

RolLs, E. T. (1992). Neurophysiological mechanisms underlying face processing within and beyond the temporal cortical visual areas. Philosophical Transactions of the Royal Society of London: Series B, 335, 11-21.

Rolls, E. T., Baylis, G. C., \& Leonard, C. M. (1985). Role of low and high spatial frequencies in the face-selective responses of neurons in the cortex in the superior temporal sulcus in the monkey. Vision Research, 25, 1021-1035.

SHASHUA, A. (1992). Illumination and view position in 3D visual recognition. In J. E. Moody, S. J. Hanson, \& R. P. Lippmann (Eds.), Advances in neural information processing systems 4 (pp. 404-411). San Mateo, CA: Morgan Kaufmann.

Shashua, A. (1996). On photometric issues in 3D visual recognition from a single 2D image. International Journal of Computer Vision, 21, 99-122.

TARR, M. J., \& Bülthoff, H. H. (1995). Is human object recognition better described by geon-structural-descriptions or by multiple-views? Journal of Experimental Psychology: Human Perception \& Performance, 21, 1494-1505.

TARr, M. J., Kersten, D., \& Bülthoff, H. H. (1998). Why the visual recognition system might encode the effects of illumination. Vision Research, 38, 2259-2275.

Tovee, M. J., Rolls, E. T., \& Azzopardi, P. (1994). Translation in- variance in the responses to faces of single neurons in the temporal visual cortical areas of the alert macaque. Journal of Neurophysiology, 72, 1049-1060.

TRoJE, N. F., \& Bülthoff, H. H. (1996). Face recognition under varying poses: The role of texture and shape. Vision Research, 36, 1761-1771.

TROJE, N. F., \& BülthoFf, H. H. (1998). How is bilateral symmetry of human faces used for recognition of novel views? Vision Research, 38, 79-89.

Ullman, S. (1996). High-level vision: Object recognition and visual cognition. Cambridge, MA: MIT Press.

WARRINGTON, E. K. (1982). Neuropsychological studies of object recognition. Philosophical Transactions of the Royal Society of London: Series $B, 298,15-33$.

WEISKRANTZ, L. (1990). Visual prototypes, memory, and the inferotemporal lobe. In E. Iwai \& M. Mishkin (Eds.), Vision, memory, and the temporal lobe (pp. 13-28). New York: Elsevier.

WILLIAMS, P., \& TARR, M. J. (1998). RSVP: Experimental control software for MacOS [On-line]. Available: http://psych.umb.edu/rsvp/

\section{NOTES}

1. Two observers had a hit rate of 1.0 , and 1 observer had a falsealarm rate of 0.0 . For these observers, $d^{\prime}$ scores were calculated from their percent correct scores, using the equation given by Macmillan and Creelman (1991) for same-different tasks.

2. Although the faces were very similar in mean luminance and RMS contrast, they were not identical.

3. Three observers failed to reach this criterion, even after given an extra practice block on the 2 nd day of the experiment. The data from these observers were excluded from the analysis. Of the remaining 17 observers, there were 5 in the cast-shadow/right-illumination condition and 4 in each of the other three conditions.

4. Trials in which the timer was incorrectly triggered were excluded from the analysis. Such trials were rare.

5. An ANOVA was performed on the median RTs raised to the $-3 / 2$ power. This transformation was necessary in order to satisfy the assumption of normality.

(Manuscript received February 1, 1998; revision accepted for publication July 30,1998 .) 